\title{
Outcomes of I-125 brachytherapy for uveal melanomas depending on irradiation dose applied to the tumor apex - a single institution study
}

\author{
Joanna Kowal, MD',2, Anna Markiewicz, MD, PhD',2, Magdalena Debicka-Kumela, MD 1,2, Anna Bogdali, MD, PhD',2, \\ Prof. Bożena Romanowska-Dixon, MD, PhDl,2 \\ 'Department of Ophthalmology and Ocular Oncology of University Hospital, Cracow, ${ }^{2}$ Department of Ophthalmology and Ocular Oncology \\ of the Jagiellonian University, Medical College, Cracow, Poland
}

\begin{abstract}
Purpose: The aim of the study was an evaluation of I-125 brachytherapy patients with uveal melanoma with special consideration for the relationship of the treatment results and the irradiation dose applied to the tumor apex.

Material and methods: Medical records of 344 adults with uveal melanoma treated with I-125 brachytherapy in the Department of Ophthalmology and Ocular Oncology of the Jagiellonian University, Medical College in Cracow, Poland were retrospectively analyzed. The study was conducted between 2003 and 2012, and the study group was divided into two subgroups depending on the irradiation dose applied to the top of the tumor: 80 Gy to 100 Gy $(n=177)$ and 100 Gy to 120 Gy $(n=167)$.

Results: It was found that the height of the tumor and the largest diameter of the tumor base decreased with every consecutive follow-up measurement and differed significantly in all comparisons $(p<0.0001)$. No significant correlation between frequency of complications was found between both study groups $\left(\chi^{2}=0.27 ; p=0.6067\right)$. The correlation between survival and the irradiation dose as applied to the tumor top was statistically irrelevant $\left(\chi^{2}=0.44 ; p=0.5084\right)$. A logistic regression model showed that patient survival depended on the largest diameter of the base and the height of tumor $(p=0.0216)$, and the risk of death was larger as these dimensions increased (IR, 1.17). An increase of the largest diameter of the base by $1 \mathrm{~mm}$ meant a $17 \%$ increase in chances of death. In $13.4 \%$ of cases, an enucleation was necessary.

Conclusions: The treatment of choroidal melanomas with I-125 iodine isotope brachytherapy is an efficient and recommended method of treatment and in many cases, an alternative to the enucleation of an eyeball.

J Contemp Brachytherapy 2018; 10, 6: 532-541 DOI: https://doi.org/10.5114/jcb.2018.79886
\end{abstract}

Key words: I-125 brachytherapy, irradiation dose, ocular melanoma, uveal melanoma.

\section{Purpose}

Uveal melanoma is the most frequent primary intra-orbital cancer in adults [1,2]. This tumor develops from melanocytes of the iris, ciliary body, or choroid, which, as a result of genetic mutation, undergo neoplastic transformation [3]. The annual prevalence of this neoplasm is estimated to be $7-8$ per $1,000,000$ persons of Caucasian race [4]. Till now, data for Poland have not been processed but it is estimated that each year there are 200-300 new cases of uveal melanoma in Poland [5]. Intra-orbital melanomas are most frequently unilateral. In $85 \%$, they develop in the choroid, in $9 \%$ in the ciliary body, and in $6 \%$ in the iris $[6,7]$.

A five-year mortality rate in case of large uveal melanomas is $53 \%$, medium size $32 \%$, and $16 \%$ in case of small tumors [8]. Uveal melanoma-related mortality is $31 \%$ by
5 years, $45 \%$ by 15 years, $49 \%$ by 25 years, and $52 \%$ by 35 years [9].

Risk factors affecting mortality include largest tumor dimension at the moment of diagnosis, tumor increase, loop-type vasculature, patient's age, tumor location (at the optic disc and/or the macula, close to vasculature, involvement of the ciliary body), epithelioid type, and extra-orbital proliferation [10]. Additionally, a genetic profile - chromosome 3 monosomy in the tumor cells, the aberrations of chromosomes 1, 3, 6, and 8 as well as mutation in GNAQ/GNA11 genes in connection with BAP1 mutation worsen the prognoses [11]. Up till now, there is no effective method of treating the metastases of uveal melanoma. Available methods, such as surgical resection (most efficient in case of individual foci located most frequently in the liver), liver chemoembolization, chemotherapy, and biological treatment may only prolong the
Address for correspondence: Anna Markiewicz, MD, PhD, Department of Ophthalmology and Ocular Oncology of the Jagiellonian University, Medical College, 38 Kopernik St., 30-150 Cracow, Poland, phone: +48 1242475 40, fax: +48 1242475 63, « e-mail: anna.markiewicz@uj.edu.pl or annamarkiewicz@interia.pl
Received: 31.05 .2018

Accepted: 08.11.2018

Published: 28.12 .2018 
survival $[12,13,14,15,16,17,18,19]$. For this reason, an early diagnosis of intra-orbital melanoma and the introduction of an appropriate local treatment is extremely important.

Local treatment methods comprise radiotherapy (teleradiotherapy and brachytherapy), surgery (enucleation, endo-, and exo-resection), transpupillary thermotherapy (TTT), and combined treatment of these methods [20,21].

As a result of the study carried out by the Collaborative Ocular Melanoma Study (COMS) group, which confirmed the same mortality rate of medium sized tumors brachytherapy and the enucleation of the eyeball (in compliance with recommendations concerning the size of the tumor), the most frequent and current method of treating uveal melanomas is radiotherapy [21]. Two methods of irradiation are applied: proton beam radiotherapy and brachytherapy. For brachytherapy, radioactive radionuclides of ruthenium $(\mathrm{Ru}-106)$, iodine (I-125), palladium (Pd-103), and iridium (Ir-192) are used [22]. In case of tumors with a size (height) larger than $5 \mathrm{~mm}, \mathrm{I}-125$ brachytherapy is most frequently used [23].

A radioactive iodine radionuclide emits gamma radiation, which is more penetrating than beta particle from ruthenium plaque and may be applied to tumors with a larger size. I-125 applicators have been applied in the Department of Ophthalmology and Ocular Oncology of the Jagiellonian University, Medical College in Cracow, Poland since 1997. The irradiation time is calculated with the plaque simulator system, which takes into consideration the tumor size (the size of the base and the height), the dose applied to the top of the tumor, and plaque activity. The recommended therapeutic dose for the top of the tumor varies between 80 and $120 \mathrm{~Gy}$. However currently, the majority of authors favor the doses calculated for the top of the tumor within the range of $80-85$ Gy $[23,24]$.

The objective of the study was to evaluate outcomes of I-125 brachytherapy treatment of patients with uveal melanoma, taking into account the relationship between results and the dose applied to the tumor apex $[25,26,27]$.

\section{Material and methods}

The study included patients with uveal melanoma treated with I-125 brachytherapy at the Department of Ophthalmology and Ocular Oncology of the Jagiellonian University, Medical College in Cracow, Poland between 2003 and 2012. The study included 344 subjects: 177 women ( $51.45 \%$ of the study group) and 167 men (48.55\%). The mean age of the subjects was $60 \pm 14$ years (ranging from 24 to 89 years). The characteristics of the study group, taking into account the gender, eye involved, the location of the anterior and posterior segment, tumor shape and color, TNM stage and the treatment type (only I-125 or I-125 in combination with transpupillary thermotherapy), and the dose applied to the apex of the tumor are presented in Table 1. The observation period varied from 3 to 144 months (medium, 53 months).

The study group was divided into two groups, with respect to the irradiation dose applied to the top of the tumor. The first group comprised of subjects with doses varied from $80 \mathrm{~Gy}$ to $100 \mathrm{~Gy}(n=177 ; 51.45 \%$ of the study group). The second group included patients with doses ranging from above $100 \mathrm{~Gy}$ to $120 \mathrm{~Gy}(n=167 ; 48.55 \%)$. The division was made on the basis of similar number of group participants, which was important for statistical analysis. At the same time, the selected doses remained within the therapeutic range.

For both groups, the application time in hours was evaluated, as displayed in Table 2 . We wanted to verify whether application times were comparable. If they were significantly different, it would influence further analysis.

The next step of the analysis was the verification of whether the average application period vary in both study groups in a statistically significant manner. Given the character of the data (the distribution, which significantly varies from the normal delivery), the Mann-Whitney $U$ test was used for the comparison.

Also, the maximum tumor height without the wall and the largest diameter of the tumor base in millimeters were determined. The tumor regression was established on the basis of comparison of obtained values describing the tumor measurement in three repeated procedures, taking into consideration irradiation dose applied to the top of the tumor. The zero hypothesis assuming the absence of any significant difference between the size of tumor for the comparable time points was verified with the Friedman test. In case of a rejection of the zero hypothesis, differences between specific measurements were verified with the Dunn test. Special attention was paid to the differences occurring after the first year of treatment.

The correlation between survival and the dose applied to the top of the tumor was analyzed and then verified with the Chi-square test.

Moreover, the frequency and type of complications divided into two groups by the irradiation doses applied to the top of the tumor were evaluated.

Patients in whose it was necessary to remove the entire eyeball within the second treatment line were evaluated according to the histopathology type of the melanoma.

The logistic regression model was verifying which of the analyzed factors (the largest base, shape, and color of the tumor) may have the biggest effect on patient survival. The study group $(n=344)$ was divided into two subgroups: the patients who survived $(n=264)$ and those who died $(n=58)$. In 22 cases, no information whether the patient was alive or not were available. These data were not included in the further analysis.

Metastases in a study group were evaluated with regards to the frequency of occurrence and the location. Statistical analysis was performed with the use of Statistica v.10 StatSoft Polska software. In all calculations, the assumed level of statistical significance was $a=0.05$. In the research, for quantitative variables, the basic statistics were calculated such as the mean, standard deviation, median, interquartile range, minimum, and maximum values. For the verification of the distribution normality, the Shapiro-Wilk test was used. In order to compare two independent groups characterized by distributions that were not normal, the Mann-Whitney test was applied. For the comparison of the three dependent groups, the Friedman analysis of variance by ranks was used. In case when obtained $p$ value allowed for questioning the null 
Table 1. A division of the number of subjects with the percentage share in the group, taking into account: tumor location, tumor shape and color, type of treatment applied, and the dose

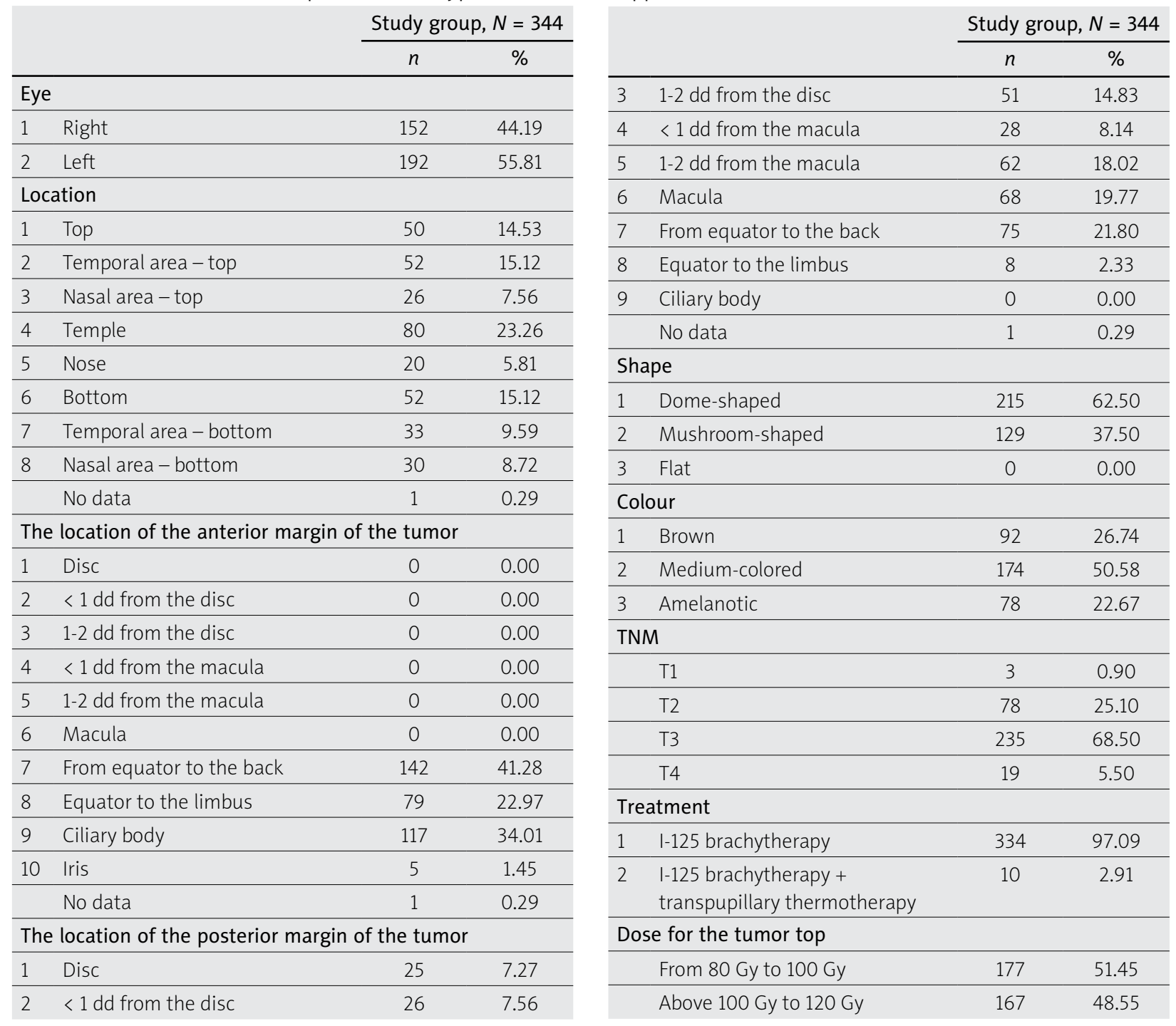

Table 2. Basic statistics defining the exposure time in hours

Application time - hours

\begin{tabular}{lllllll}
\hline $\begin{array}{l}\text { Irradiation dose to the tumor } \\
\text { top }\end{array}$ & Mean & SD & Median & Min & Max & IQR \\
\hline 80 Gy to 100 Gy & 82.01 & 35.24 & 72.00 & 24.50 & 193.60 & $51.50-98.50$ \\
\hline Above 100 Gy to 120 Gy & 82.03 & 33.54 & 69.50 & 28.50 & 171.00 & $66.00-95.65$ \\
\hline
\end{tabular}

hypothesis, which assumed the equality of median values of studied property in consecutive measurements, additional post-hoc tests for Friedman test were performed, with the use of Dunn test. In such a way, it was explained which groups differed from each other. For verification of correlations between studied variables, the Chi-square test or the accurate Fischer test were performed.

Authors declare that this investigation was carried out following the rules of the Declaration of Helsinki from 1975 (revised in 2008).

\section{Results}

\section{The irradiation exposure period for both dose groups}

In the analysis of the application time for both groups, the obtained value was $p=0.3787(U=0.88)$, which did not point to the presence of statistically significant differences between the application time in the group of subjects with doses ranging from 80 to $100 \mathrm{~Gy}$ and the group with doses from 100 to 120 Gy (Table 2). 


\section{The height and the largest diameter of the tumor base for both dose groups}

The evaluation of the height and the largest diameter of the tumor base, performed in three time points: before the treatment, after the first year of treatment, and at the last follow-up visit, showed that the tumor size was not the same within the studied time period.

The comparison of the values $p<0.0001$ obtained within Friedman test, with the significance level $\alpha=0.05$ allowed to conclude that the tumor size differed significantly in all the studied comparisons: 1 . The size before the treatment and the size after the first year of treatment $(p<0.0001) ; 2$. The size before the treatment and the size at the last follow-up visit $(p<0.0001) ; 3$. The size after the first year of treatment and the size at the last follow-up visit $(p<0.0001)$.

The tumor size was smaller with every consecutive measurement. This conclusion applied both to the maxi- mum tumor height without the wall and the largest base diameter, taking into account the irradiation dose calculated per the tumor top for both groups (lower and higher dose). The obtained data are presented in Figures 1 and 2.

Detailed data concerning the treatment efficiency showed that after one year of treatment, tumor regression was observed in $89.83 \%$ patients, in $8.43 \%$ a recurrence was diagnosed, in $0.58 \%$ there was no reaction to the treatment and in $1.16 \%$, no data were available with regards to the fact that patients failed to turn up for follow-up visit (Figure 3).

Additionally, tumor condition after one year of treatment was presented, divided into the doses applied to the tumor top. The results are presented in Table 3. Considering the expected values and the observed values, the correlation between tumor condition after the first year (regression/recurrence/no reaction) and the irradiation dose applied to the tumor top was verified with the
A

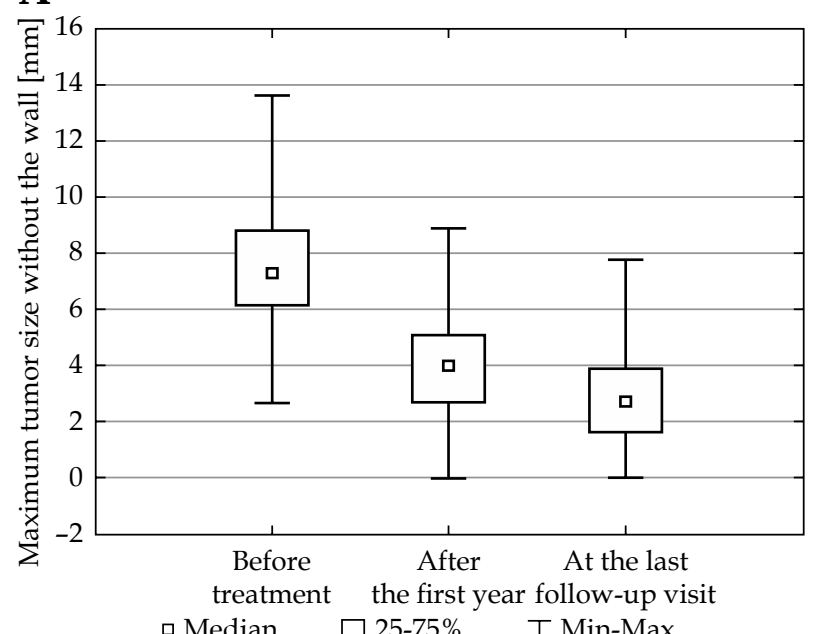

B

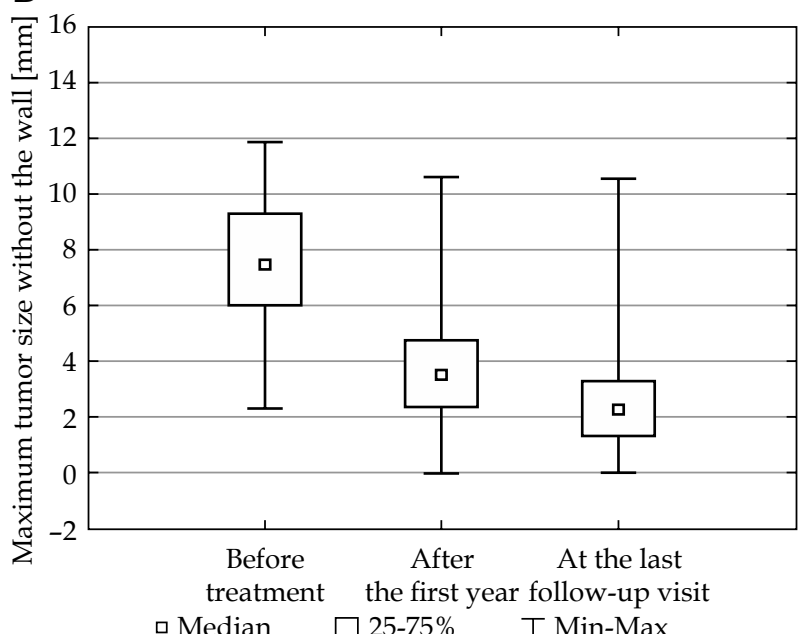

Fig. 1. Maximum tumor height without the wall measured in three time-points: for the patient groups with the irradiation dose applied to the tumor top from 80 to $100 \mathrm{~Gy}$ (A) and from above 100 to $120 \mathrm{~Gy}$ (B) (ANOVA FRIEDMAN RANK test; $p<0.0001$ )

A

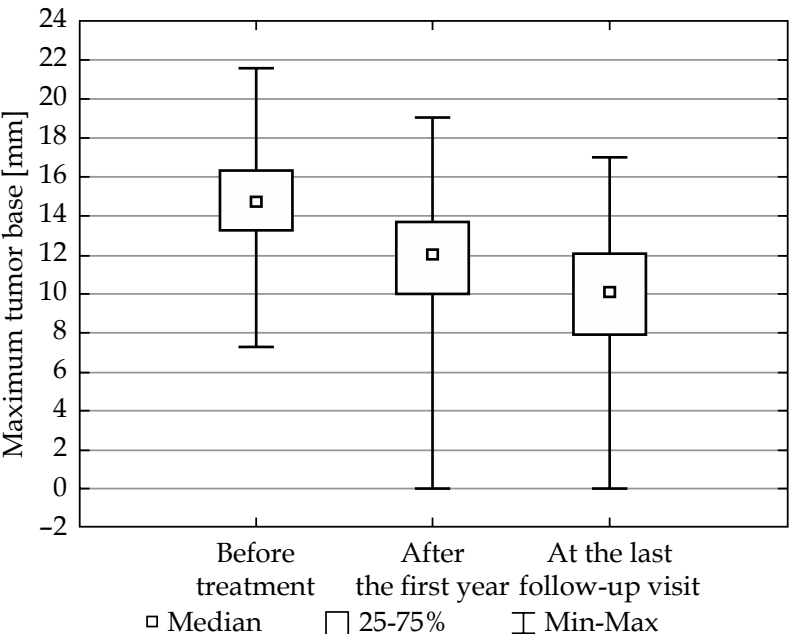

B

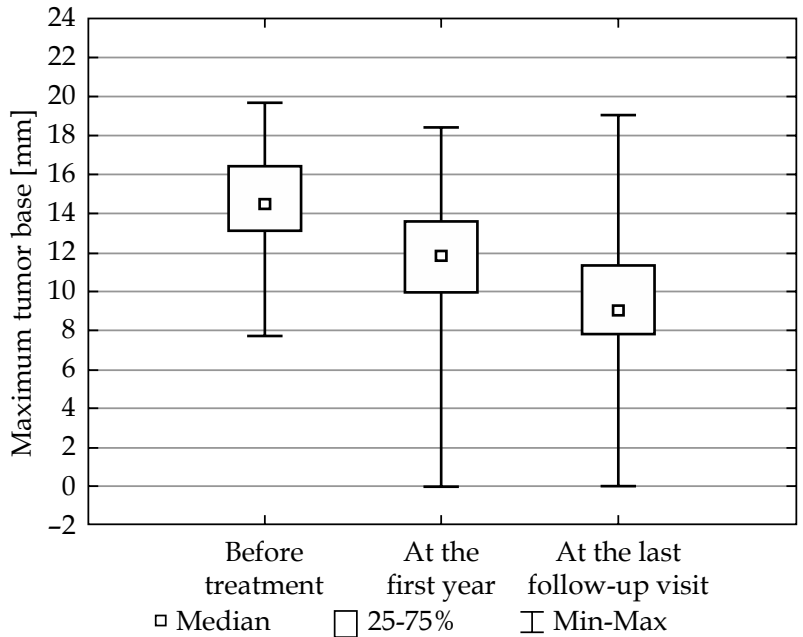

Fig. 2. The largest size of the tumor base measured in three time points for the groups of patients from 80 to $100 \mathrm{~Gy}$ (A) and from above $100 \mathrm{~Gy}$ to $120 \mathrm{~Gy}$ (B) (ANOVA FRIEDMAN RANK test; $p<0.0001$ ) 

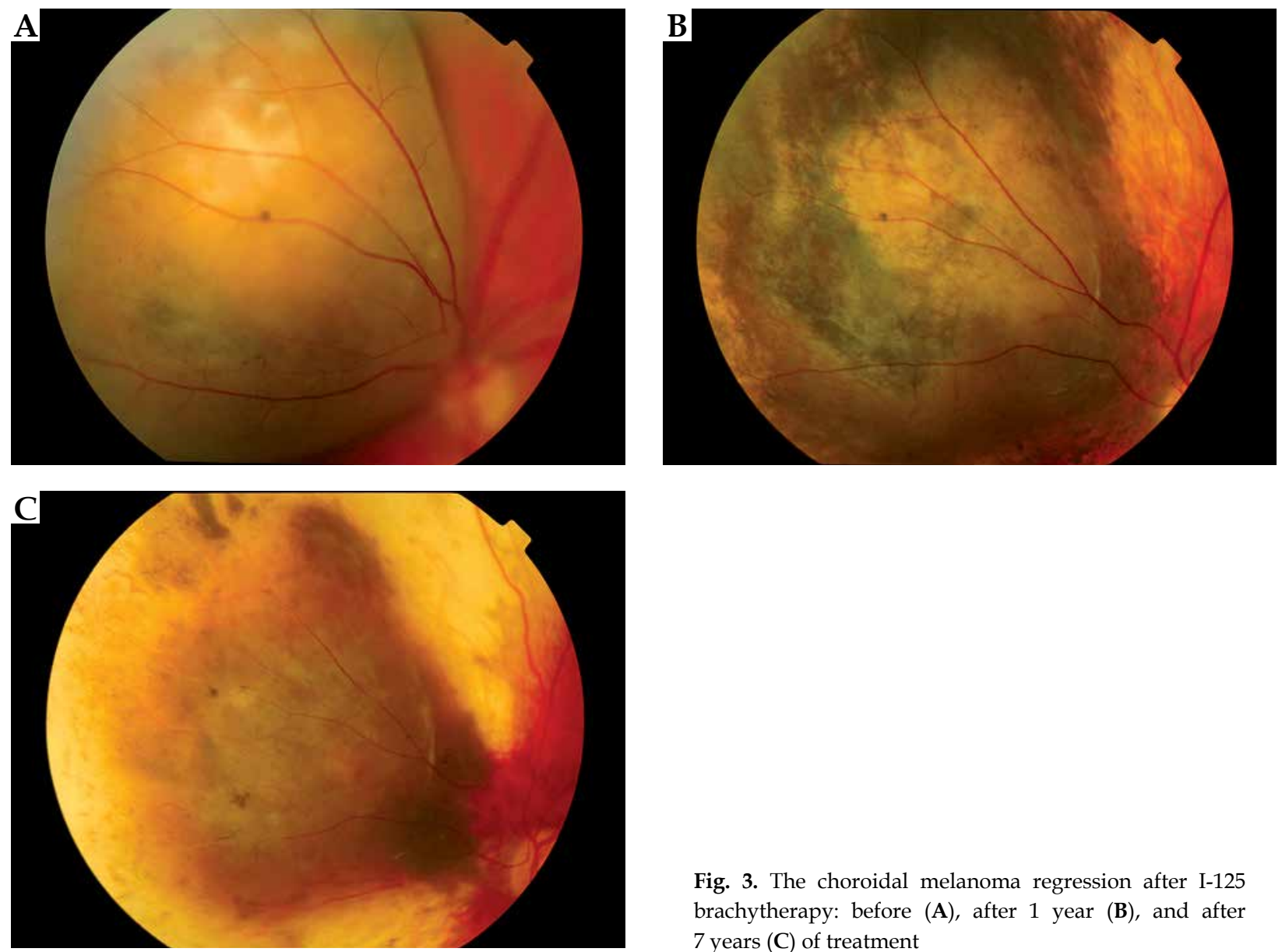

Fig. 3. The choroidal melanoma regression after I-125 brachytherapy: before (A), after 1 year (B), and after 7 years $(\mathbf{C})$ of treatment

Table 3. The tumor condition in the first year of treatment in general and divided into subgroups with respect to the dose applied to the top of tumor

\begin{tabular}{lccccccc} 
Tumour condition & \multicolumn{2}{c}{ Entire group } & \multicolumn{2}{c}{80 Gy to 100 Gy } & \multicolumn{2}{c}{ Above 100 to 120 Gy } \\
\cline { 2 - 7 } & $n$ & {$[\%]$} & $n$ & {$[\%]$} & $n$ & {$[\%]$} & 87.43 \\
\hline Regression & 309 & 89.83 & 163 & 92.09 & 146 & 18 & 10.78 \\
\hline Recurrence & 29 & 8.43 & 11 & 0.56 & 1 & 0.60 \\
\hline No reaction & 2 & 0.58 & 1 & 1.13 & 2 & 1.20
\end{tabular}

Fischer's exact test. The obtained value $p=0.2523 \mathrm{did}$ not prove to reject the zero hypothesis of no correlation between the studied variables. In the first year, regression was observed in $92 \%$ of cases in the group with a dose from 80 Gy to $100 \mathrm{~Gy}$ and $87 \%$ in the group with a dose from $100 \mathrm{~Gy}$ to $120 \mathrm{~Gy}$.

\section{Complications for both dose groups}

The frequency of complications was evaluated with respect to both groups analyzed. In case of patients with the irradiation dose applied to the tumor top from 80 Gy to 100 Gy $(n=177)$, complications were found in 170 subjects (95.05\% of the study group). Only 7 persons (3.95\%) did not report any treatment-related complications. In the patient group with irradiation dose from $100 \mathrm{~Gy}$ to $120 \mathrm{~Gy}(n=167)$, complications were observed in 163 subjects $(97.60 \%$ of the study group). Only 4 persons $(2.40 \%)$ did not report any treatment-related complications. The Chi-square test with the Yates correction was used to verify whether there was any correlation between the occurrence of complications and the irradiation dose applied to the tumor top. The obtained results $\left(\chi^{2}=0.27 ; p=0.6067\right)$ did not reveal the presence of the suspected correlation. It was worth pointing out that the group of patients with complications $(n=333)$ was much larger than the group of patients without complications $(n=11)$, which significantly affected obtained results.

Table 4 presents the types of complications and their numbers as compared to the dose applied to the tumor top. In many patients, there were numerous complications 
Table 4. Analysis of the complications in the groups of doses applied to the tumor top: from 80 Gy to 100 Gy and above 100 Gy to 120 Gy

Irradiation dose applied to the tumor top:

$$
\text { From } 80 \text { to } 100 \text { Gy }(n=177)
$$

Above 100 to 120 Gy $(n=167)$

\begin{tabular}{|c|c|c|c|c|}
\hline Existing complications & $n$ & [\%] & $n$ & [\%] \\
\hline Rubeosis of the iris & 42 & 10.88 & 46 & 12.14 \\
\hline Secondary glaucoma & 53 & 13.73 & 57 & 15.04 \\
\hline Cataract & 155 & 40.16 & 154 & 40.63 \\
\hline Maculopathy & 8 & 2.07 & 4 & 1.06 \\
\hline Neuropathy & 14 & 3.63 & 12 & 3.17 \\
\hline Retinopathy & 48 & 12.44 & 37 & 9.76 \\
\hline Hemophthalmias & 24 & 6.22 & 19 & 5.01 \\
\hline Retinal detachment & 13 & 3.37 & 13 & 3.43 \\
\hline Scleromalacia & 2 & 0.52 & 3 & 0.79 \\
\hline Posterior adhesions & 27 & 6.99 & 34 & 8.97 \\
\hline Total & 386 & 100 & 379 & 100 \\
\hline
\end{tabular}

(more than 1); the amount of the complications reported was higher than the number of patients in the study.

The most frequently occurring complication in both patient groups analyzed was cataracts (more than $40 \%$ of existing complications), with secondary glaucoma as the another most frequent complication. Scleromalacia was the least frequently occurring complication (less than $1 \%$ in both dose groups).

\section{Removal of the eyeball (enucleation) as the next (the second) line of treatment depending on the irradiation dose applied to the tumor apex during primary brachytherapy}

In case of 46 patients ( $13.37 \%$ of the study group), it was necessary to remove an eyeball. In 15 cases, this was the outcome of the recurrence of the tumor, and in 31 cases it was caused by complications and resulting pain. Among removed eyeballs, a mixed type of melanoma was dominating $(41.30 \%)$, followed by epithelioid $(23.90 \%)$, necrotic $(17.40 \%)$, and spindle-cell type $(13.00 \%)$, whilst in $4.40 \%$ of cases no data were available.

The mixed type $(60.00 \%)$ was dominating among 15 removed eyeballs due to local recurrence, followed by epithelioid (20.00\%), and necrotic $(20.00 \%)$.

The results of the Chi-square test $\left(x^{2}=0.08 ; p=0.7463\right)$ did not show any correlation between the occurrence of enucleation as the second line treatment and the irradiation dose applied to the tumor top during the primary treatment.

\section{Evaluation of the occurrence of distant metastases and the analysis of their location for both dose groups}

Metastases occurred in 29 subjects, making up $8.43 \%$ of the study group. In case of 10 patients $(2.91 \%)$, no data were available. The next study step was to evaluate the location of the metastases. Data concerning the location of the distant metastases in 29 patients are presented in Table 5.

The category of "other" includes one patient, in whose case metastases were observed in femur. Three patients, apart from metastases in the liver, also had metastases located in the adrenal glands ( 1 person), lungs ( 1 person), and minor pelvis ( 1 person). The distant metastases of melanoma were most frequently located in the liver.

Additionally, the occurrence of metastases was divided depending on the irradiation dose applied to the tumor top. The numbers of percentages are presented in Table 6 . The results of the Chi-square test $\left(\chi^{2}=0.09 ; p=0.7675\right)$ did not show any correlation between the occurrence of metastases and irradiation dose applied to the tumor top.

\section{Survival depending on the irradiation dose applied to the tumor apex}

The Chi-square test was used to verify the existence of some correlations between survival and irradiation dose applied to the tumor top. The obtained results $\left(\chi^{2}=0.44\right.$; $p=0.5084$ ) did not show any existence of suspected correlations (Table 7).

The constructed model of logistic regression allowed to draw the following conclusions:

1. Patient survival significantly depends on the size of the largest diameter of the tumor base $(p=0.0216)$;

Table 5. The location of the metastases in patients $(n=29)$

\begin{tabular}{lcc} 
Metastases location & $n$ & {$[\%]$} \\
\hline Liver & 24 & 82.76 \\
\hline Lung & 1 & 3.45 \\
\hline Liver + other locations & 3 & 10.34 \\
\hline Other locations & 1 & 3.45
\end{tabular}


Table 6. The occurrence of metastases in the study group $(n=344)$ and divided into 2 subgroups depending on the dose applied to the tumor top

\begin{tabular}{lccccccc} 
Metastases & \multicolumn{2}{c}{ Whole group of patients } & \multicolumn{2}{c}{80 Gy to 100 Gy } & \multicolumn{2}{c}{ Above 100 to 120 Gy } \\
\cline { 2 - 8 } & $n$ & {$[\%]$} & $n$ & {$[\%]$} & $n$ & 149 & 89.22 \\
\hline Absent & 305 & 88.66 & 156 & 14 & 7.91 & 15 & 8.98 \\
\hline Present & 29 & 8.43 & 7 & 3.95 & 3 & 1.80
\end{tabular}

Table 7. Patient survival with respect to the irradiation dose applied to the tumor top

\begin{tabular}{lcccc} 
Dose & \multicolumn{2}{c}{80 Gy to 100 Gy } & \multicolumn{2}{c}{ Above 100 Gy to 120 Gy } \\
\hline Condition & $n$ & {$[\%]$} & $n$ & [\%] \\
\hline Patient is alive & 133 & 75.14 & 131 & 26.44 \\
\hline Death & 32 & 18.08 & 10 & 15.57 \\
\hline No data & 12 & 6.78 & & 5.99
\end{tabular}

Table 8. Survival depending on the largest base diameter, tumors shape, and color

\begin{tabular}{lcccccc} 
& Evaluation & Statistic error. & $\chi^{2}$ Wald & $p$ value & Odds ratio (OR) & $95 \% \mathrm{Cl}$ for OR \\
\hline Constant term & -4.13 & 1.35 & 9.32 & 0.0023 & 0.02 & $0.001-0.23$ \\
\hline Maximum tumor base & 0.16 & 0.07 & 5.28 & 0.0216 & 1.17 & $1.02-1.34$ \\
\hline Tumor shape & 0.60 & 0.33 & 3.32 & 0.0683 & 1.82 & $0.95-3.46$ \\
\hline Tumor color & -0.30 & 0.21 & 1.95 & 0.1625 & 0.74 & $0.49-1.13$
\end{tabular}

2. The risk of death is higher with an increase of the size of the diameter (odds ratio, 1.17); 3 . With an increase in the largest diameter of the tumor base by $1 \mathrm{~mm}$, the probability of death increases by $17 \% ; 4$. No significant influence of the tumor shape and color on the occurrence of death was found.

The analysis revealed that the constructed model was statistically significant, which was evidenced by the result of the likelihood ratio test $\left(\chi^{2}=8.49 ; p=0.0369\right)$. A sufficient quality of model fitting was confirmed by the result of the Hosmer-Lemeshow test $(p=0.2314)$. All data are presented in Table 8 .

Given the fact that the largest diameter of the tumor base and its height were correlated with each other, only the tumor base was included in the logistic regression model. The calculated Spearman's rank correlation coefficient was $r=0.15$, which signifies a very weak correlation, yet was still statistically significant $(p=0.0066)$; therefore, the authors decided to include only the largest tumor diameter into the logistic regression model.

\section{Discussion}

In 2006, after COMS revealed the results of medium-sized uveal melanomas treatment, consisting of irradiation with the I-125 radionuclide as opposed to the removal of an eyeball (pointing to the same length of patients' survival in both groups), a conservative treatment with brachytherapy became the treatment standard $[21,24]$.
Since 1968, patients in our Department have been treated with Co-60 and currently, two types of radionuclide plaques have been used. The first one has contains ruthenium (Ru-106), used since 1996, and the second includes iodine (I-125), since 1997. The applicators containing I-125 are used in cases of tumors where height is equal to or larger than $5 \mathrm{~mm}$, but not larger than $10 \mathrm{~mm}$, with a base diameter not larger than $18 \mathrm{~mm}$. So far, there have been more than 1,900 patients treated with iodine brachytherapy. This was the reason why the authors decided to analyze the results of 10-year treatment period using I-125, with special attention paid to irradiation dose applied to the tumor top. The patients were divided into two groups: one group receiving dose from 80 to $100 \mathrm{~Gy}$ to the top of the tumor and the other from above 100 to 120 Gy. The application time period was comparable in both groups $(p=0.3787)$ and medium was 82.01 hours for the group from 80 to $100 \mathrm{~Gy}$, and medium 82.03 hours for the group above 100 to $120 \mathrm{~Gy}$. These time periods are compliant with the recommendations of COMS and the American Brachytherapy Society [21,23].

In the current study, the analyzed decrease in height of tumor and the largest diameter of the tumor base in three time-points: before treatment, after one year of treatment, and at the last follow-up visit showed that the size of tumor was not the same within the analyzed time period and that these size differences were statistically significant $(p<0.0001)$ in all cases. In the selected two dose groups, in a significant majority of cases, the tumor 
height measured (not considering the eyeball wall) as well as the largest tumor diameter decreased $(p<0.0001)$. In the COMS studies carried out by Demirci et al. and Garcia-Alvarez et al., the decrease of the size of the uveal melanoma was on a similar level $[24,25,28,29]$. Additionally, Demirci et al. observed that the tumor regression after the treatment of tumors with a larger baseline height is larger than in the case of tumors with a smaller height, especially in the initial observation period [28]. In our study we also noticed a quicker diminishing of the tumor height in the initial period of the observation.

Similarly, in the evaluation of the influence of the dose applied to the height of tumor and tumor condition in categories such as regression, recurrence, and lack of any reaction, during the first year of treatment, no statistical difference was observed in both our study groups with irradiation doses from 80 Gy to $100 \mathrm{~Gy}$ and from $100 \mathrm{~Gy}$ to $120 \mathrm{~Gy}$ (Fischer's exact test, $p=0.2523$ ). The regression was observed in $92 \%$ and $87 \%$ cases, respectively. Rashid et al. when creating regression models of uveal melanomas after brachytherapy: Ru-106 median apex dose was 102 Gy (range, 62-194 Gy), and I-125 median apex dose was 80 Gy (range, 49-164 Gy), did not observe influence of the level dose on tumor regression [30].

In the opinion of some authors, the increase of the dose applied to the top of the tumor leads to an increase of complications risk [22,25,31,32,33]. Among our patients, in the group with an irradiation dose from $80 \mathrm{~Gy}$ to 100 Gy $(n=177)$, complications were diagnosed in 170 cases $(95.05 \%$ of the study group) and, in the group with the irradiation dose from 100 Gy to 120 Gy $(n=167)$, complications occurred in 163 of cases $(97.60 \%$ of the study group). Only 4 patients (2.40\%) from the first group and 7 patients $(3.95 \%)$ from the second did not have any treatment-related complications. In the first group, the number of post-irradiation complications was lower than in the second group. The obtained results $\left(\chi^{2}=0.27\right.$; $p=0.6067)$ did not reveal the presence of suspected correlations. It was worth pointing out that the group of patients with complications $(n=333)$ was much larger than the group of patients without complications $(n=11)$, which significantly affected the results obtained. Hence, a lack of significant relation between the increase in the level of dose and the increase in the number of complications in our study can be concluded. The complications occurring after I-125 brachytherapy are frequent and their number increases with the follow-up period. The results, which we obtained are comparable with observations of other authors and COMS [31,32,33]. The results obtained in specific centers may differ and they depend on the location of the applicator (e.g., paracentral in order to spare the disc or the macula), and also on possibility of designing the system of sources in the applicator as well as on selection of its individual shape and size for a patient, which decreases the number of complications [34]. A significant factor, which affects a decrease in the number of complications and presence of local recurrence is the intraoperative, ultrasound confirmation of the location of the applicator in tumor base $[35,36,37]$.
In the opinion of the American Brachytherapy Society and COMS protocol, in I-125 brachytherapy, the recommended irradiation dose of $85 \mathrm{~Gy}$ applied to the top of tumor is efficient and gives the least number of complications $[23,24]$.

In our study, metastases were found in 29 patients ( $8.43 \%$ of the study group), whilst in case of 10 patients $(2.91 \%)$, no data were available. No statistically significant difference in the frequency of metastases in the two studied groups were observed. Wagner et al., on the basis of analysis of 88 cases found that small doses applied to the tumor top are connected with a larger risk of distant metastases [26]. In our study, the smallest irradiation dose applied to the tumor top was $80 \mathrm{~Gy}$, which fits into the recommended therapeutic scopes, whilst an increase of this dose (even to $120 \mathrm{~Gy}$ ) did not have any influence on occurrence. These differences could result from a smaller number of analyzed patient's group, and this group mainly involved patients with low-stage of disease (T1a to T2a, cover $80 \%$ ) in Wagner et al.'s study, which influences the obtained outcomes.

A local recurrence of melanoma occurred in $8.43 \%$ of patients after 1-year of treatment. Enucleation of the eye for this reason was performed in $4.5 \%$ cases. In $9.0 \%$ of patients, enucleation was necessary with regards to the pain resulting from post-irradiation complications. The obtained results are comparable with those reported by other authors [29,38]. Jampol et al. concluded that local failures in treatment constitute the most frequent cause of enucleation within the first 3 years of treatment, whereas after 3 years, the complications are the most common reason. Additionally, the risk of the eyeball removal within 5 years is $12.5 \%$, and risk of recurrence is $10.3 \%$. In the opinion of COMS, the probability of recurrence and enucleation after the treatment with I- 125 is low but it is connected with poorer prognosis [31]. The meta-analysis performed by Chang et al. of 49 articles (12,524 patients) showed that the risk of local recurrence in treatment with I-125 varies between $4.0 \%$ and $9.6 \%$ [37].

With the current stage of knowledge, the analysis of the factors affecting the treatment of uveal melanoma with brachytherapy must also include genetic factors. Shields et al. showed that the tumor regression (between 12 and 15 months after the treatment) is faster in the melanoma with monosomy of chromosome 3 , than in case of a disomy [39]. Similar conclusions were drawn by Marathe et al. [40].

The Chi-square test was used to verify the existence of correlation between survival and the dose applied to the top of tumor. The results $\left(\chi^{2}=0.44 ; p=0.5084\right)$ did not prove the existence of studied correlation. Wagner et al. in their work observed that small doses applied to the tumor top lead to a large risk of metastases, which is related to a lower survival rate. In the above study, the average dose applied to the tumor top was $85.90 \mathrm{~Gy}$ (with the scope ranging from $83.72 \mathrm{~Gy}$ to $147.20 \mathrm{~Gy}$ ) [26]. In turn, for $\mathrm{Ru}-106$, the risk of local recurrence was not associated with the prescribed doses during brachytherapy or time of irradiation $[41,42,43]$. The local recurrence increases the risk of metastasis by a hazard ratio (HR) of 6.28 
(95\% CI: 4.4-8.9; $p<0.001)$ and it is associated with poorer survival $[41,44]$. The systematic review of fifteen retrospective and prospective studies with I-125 treatment demonstrated a 1.0 Gy increase in medium study dose associated with a $0.14 \%$ decrease in local recurrence rate, which was not statistically significant $(p=0.336)$. Ranges of reported mean or median radiation dose to tumor apex were 62.5-104.0 Gy. Local recurrence rates ranged from 0 to $24 \%$ [27].

In the studied group of patients, the influence of the height of tumor, the largest base diameter, the tumor shape and color on the patient survival for both dose group were also analyzed. A construction of the model of logistic regression allowed to conclude that patient survival depends significantly on the size of the largest tumors base diameter $(p=0.0216)$ and with its increase, the risk of death is higher (odds ratio, 1.17), which means that with an increase of the largest tumor base diameter by $1 \mathrm{~mm}$, the probability of death is $17 \%$ greater; no statistical difference was observed in both our study groups. With regards to the fact that the largest tumor base diameter and the tumor height were correlated with each other, only the measurement of the tumor base was included into the model of logistic regression (Spearman's rank correlation was $r=0.15)$, which means that this correlation is statistically significant $(p=0.0066)$; therefore, these conclusions may also apply to the height of tumor.

Conclusions regarding the significant influence of the tumor base diameter on patients' survival were also drawn by the team of Damato et al. $(p<0.001)$ who analyzed the tumors in three categories determined by the size of the tumor base (<10 mm, 10-15 mm, and > $15 \mathrm{~mm})$ [10,45] Shields et al. analyzed 8,033 eyeballs with the uveal melanoma and found that one of the most significant features in the evaluation of the prognosis was the tumor dimensions: both the largest base diameter and the tumor height. Their findings were that an increase of the tumor height by $1 \mathrm{~mm}$ results in an increase of the probability of death by $6 \%$ (hazard ratio, 1.06) and that during a 10-year observation period, the risk of distant metastases of the primary tumors with a thickness of $0-1.0 \mathrm{~mm}$ is $6 \%$, whereas in case of tumors with a size (height) above $10 \mathrm{~mm}$, this risk increases to $51 \%$ [7]. By competing risks regression analysis, the hazard ratio in an increase of the probability of death was $1.08(p=0.0012)$ for each millimeter increase in tumor diameter in Kujala et al.'s study [9].

\section{Conclusions}

The treatment of choroidal melanomas with brachytherapy with the I-125 iodine radionuclide is an efficient and recommended method of treatment, and in many cases, an alternative to the enucleation of an eyeball. In our study, no significant differences between both dose groups (low 80-100 Gy and high 100-120 Gy) in the influence of tumor regression, number of complications, metastasis appearing, number of removed eyeballs (in a second line treatment), and patient survival were found. Moreover, the biggest decrease in the tumor size appeared in the first year after I-125 treatment. We recommend conducting further studies to evaluate the in- fluence of I-125 prescribed dose on the tumor apex and the final treatment outcomes, taking into account other prognostic factors. In our opinion, a randomized study would be the best option.

\section{Disclosure}

Authors report no conflict of interest.

\section{References}

1. Kaliki S, Shields CL. Uveal melanoma: relatively rare but deadly cancer. Eye 2017; 31: 241-257.

2. Shields JA, Shields CL. Intraocular Tumors. WB Saunders Co, Philadelphia 1992; 25-43, 51-59, 66-77, 118-136, 156-169, 171-205.

3. Proniewska-Skrętek E, Wojnar M, Mariak Z, Zalewska R. Genetic abnormalities underlying formation of choroidal melanoma. Klin Oczna 2005; 107: 140-142.

4. Isager P, Ehlers N, Overgaard J. Have choroidal and ciliary body melanomas changed during the period 1955-2000? Acta Ophtalmol Scand 2004; 82: 509-516.

5. Romanowska-Dixon B, Pogrzebielski A, Bogdali A et al. Proton beam radiotherapy of uveal melanoma - preliminary results. Klin Oczna 2012; 114: 173-179.

6. Żygulska-Mach H. Epidemiologia czerniaka oka. Wspótcz Onkol 1998; 2: 226-227 [in Polish].

7. Shields CL, Furuta M, Thangappan A et al. Metastasis of uveal melanoma millimeter-by-millimeter in 8033 consecutive eyes. Arch Ophthalmol 2009; 127: 989-998.

8. Diener-West M, Hawkins BS, Markowitz JM, Schachat AP. A review of mortality from choriodal melanoma, II: meta-analysis of 5-year mortality rates following enucleation, 1966 through 1988. Arch Ophtalmol 1992; 110: 245-250.

9. Kujala E, Mäkitie T, Kivelä T. Very long-term prognosis of patients with malignant uveal melanoma. Invest Ophthalmol Vis Sci 2003; 11: 4651-4659.

10. Damato B, Eleuteri A, Taktak AF, Coupland SE. Estimating prognosis for survival after treatment of choroidal melanoma. Prog Retin Eye Res 2011; 30: 285-295.

11. Gill HS, Char DH. Uveal melanoma prognostication: from lesion size and cell type to molecular class. Can J Ophthalmol 2012; 47: 246-253.

12. Damato B, Coupland SE. Translating uveal melanoma cytogenetics into clinical care. Arch Ophthalmol 2009; 127: 423-429.

13. Triozzi PL, Singh AD. Adjuvant therapy of uveal melanoma: current status. Ocul Oncol Pathol 2014; 1: 54-62.

14. Weis E, Salopek TG, McKinnon JG et al. Management of uveal melanoma: a consensus-based provincial clinical practice guideline. Curr Oncol 2016; 23: e57-e64.

15. Mariani P, Piperno-Neumann S, Servois V et al. Surgical management of liver metastases from uveal melanoma: 16 years' experience at the Institut Curie. Eur J Surg Oncol 2009; 35: 1192-1197.

16. Gonsalves CF, Eschelman DJ, Thornburg B et al. Uveal melanoma metastatic to the liver: Chemoembolization with 1,3-bis-(2-chloroethyl)-1-nitrosourea. AJR Am J Roentgenol 2015; 205: 429-433.

17. Carling U, Dorenberg EJ, Haugvik SP et al. Transarterial chemoembolization of liver metastases from uveal melanoma using irinotecan-loaded beads: treatment response and complications. Cardiovasc Intervent Radiol 2015; 38: 1532-1541.

18. Valsecchi ME, Terai M, Eschelman DJ et al. Double-blinded, randomized phase II study using embolization with or without granulocyte-macrophage colony-stimulating factor in uveal melanoma with hepatic metastases. J Vasc Interv Radiol 2015; 26: 523-532.e2. 
19. Oliva M, Rullan AJ, Piulats JM. Uveal melanoma as a target for immune-therapy. Ann Transl Med 2016; 4: 172.

20. Rao HJ, Sein J, Badiyan S et al. Patterns of care and survival outcomes after treatment for uveal melanoma in the postcoms era (2004-2013): a surveillance, epidemiology, and end results analysis. J Contemp Brachytherapy 2017; 9: 453-465.

21. Diener-West M, Earle JD, Fine SL et al.; Collaborative Ocular Melanoma Study Group. The COMS randomized trial of iodine 125 brachytherapy for choroidal melanoma, III: initial mortality findings. COMS Report No. 18. Arch Ophthalmol 2001; 119: 969-982.

22. Leonard KL, Gagne NL, Mignano JE et al. A 17-year retrospective study of institutional results for eye plaque brachytherapy of uveal melanoma using (125) I, (103) Pd and (131) Cs and historical perspective. Brachytherapy 2011; 10: 331-339.

23. The American Brachytherapy Society - Ophthalmic Oncology Task Force. The American Brachytherapy Society consensus guidelines for plaque brachytherapy of uveal melanoma and retinoblastoma. Brachytherapy 2014; 13: 1-14.

24. Collaborative Ocular Melanoma Study Group. The COMS randomize trial of iodine 125 brachytherapy for choroidal melanoma: V. Twelve-year mortality rates and prognostic factors: COMS report No. 28. Arch Ophthalmol 2006; 124: 1684-1693.

25. Miguel D, de Frutos-Baraja JM, Lopez-Lara F et al. Visual outcome after posterior uveal melanoma episcleral brachytherapy including radiobiological doses. J Contemp Brachytherapy 2018; 10: 123-131.

26. Wagner A, Chen A, Cook T et al. Outcomes and Control Rates for I-125 Plaque Brachytherapy for Uveal Melanoma: A Community-Based Institutional Experience. ISRN Ophthalmol 2014; 2014: 950975.

27. Echegaray JJ, Bechrakis NE, Singh N et al. Iodine-125 brachytherapy for uveal melanoma: a systematic review of radiation dose. Ocul Oncol Pathol 2017; 3: 193-198.

28. Demirci H, Saponara F, Khan A et al. Regression rate of posterior uveal melanomas following Iodine- 125 plaque radiotherapy. Afr J Ophthalmol 2015; 22: 103-107.

29. Garcia-Álvarez C, Saornil MA, López-Lara F et al. Episcleral brachytherapy for uveal melanoma: analysis of 136 cases. Clin Transl Oncol 2012; 14: 350-355.

30. Rashid M, Heikkonen J, Kivelä T. Tumor regression after brachytherapy for choroidal melanoma: reduction of thickness and cross sectional area by shape and regression pattern. Invest Ophthalmol Vis Sci 2015; 56: 2612-2623.

31. Jampol LM, Moy CS, Murray TG et al. For the COMS Follow-up of Plaque Eyes Working Group: The COMS Randomized Trial of Iodine 125 Brachytherapy for Choroidal Melanoma IV. Local Treatment Failure and Enucleation in the First 5 Years after Brachytherapy, COMS Report No. 19. Ophthalmology 2002; 109: 2197-2206.

32. Szuścik I, Romanowska-Dixon B, Markiewicz A et al. Neuropatia popromienna po brachyterapii czerniaka naczyniówki. (Radiation optic neuropathy after brachytherapy of uveal melanoma). Klin Oczna 2006; 108: 278-280.

33. Shields CL, Naseripour M, Cater J et al. Plaque radiotherapy for large posterior uveal melanomas ( $>$ or $=8-\mathrm{mm}$ thick) in 354 consecutive patients. Ophthalmology 2002; 109: 1838-1849.

34. Gagne NL, Cutright DR, Rivard MJ. Keeping an eye on the ring: COMS plaque loading optimization for improved dose conformity and homogeneity. J Contemp Brachytherapy 2012; 4: $165-175$.

35. Wen JC, Oliver SC, McCannel TA. Ocular complications following I-125 brachytherapy for choroidal melanoma. Eye 2009; 23: 1254-1268.
36. Chang MY, Kamrava M, Demanes DJ et al. Intraoperative ultrasonography-guided positioning of Iodine 125 plaque brachytherapy in the treatment of choroidal melanoma. Ophthalmology 2012; 119: 1073-1077.

37. Chang MY, McCannel TA. Local treatment failure after globe-conserving therapy for choroidal melanoma. $\mathrm{Br} \mathrm{J} \mathrm{Oph-}$ thalmol 2013; 97: 804-811.

38. Caminal Mitjana JM, Quintana Casany M, Pera Fábregas J et al. Results of Iodine-125 radiotherapy in the treatment of uveal melanoma. Arch Soc Esp Oftalmol 2002; 77: 29-38.

39. Shields CL, Bianciotto C, Rudich D et al. Regression of uveal melanoma after plaque radiotherapy and thermotherapy based on chromosome 3 status. Retina 2008; 28: 1289-1295.

40. Marathe OS, Wu J, Lee SP et al. Ocular response of choroidal melanoma with monosomy 3 versus disomy 3 after Iodine-125 brachytherapy. Int J Radiat Oncol Biol Phys 2011; 81: 1046-1048.

41. Respond-Kubiak I, Wróblewska-Zierhoffer M, TwardoszPawlik $\mathrm{H}$ et al. Ruthenium brachytherapy for uveal melanoma - single institution experience. J Contemp Brachytherapy 2017; 9: 548-552

42. Damato B, Patel I, Campbell IR et al. Local tumor control after $106 \mathrm{Ru}$ brachytherapy of choroidal melanoma. Int J Radiat Oncol Biol 2005; 63: 385-391.

43. Rouberol F, Roy P, Kodjikian L et al. Survival, anatomy, and functional long-term results in choroidal and ciliary body melanoma after ruthenium brachytherapy (15 years' experience with beta-rays). Ophthalmology 2004; 137: 893-900.

44. Ophthalmic Oncology Task Force. Local recurrence significantly increases the risk of metastatic uveal melanoma. Ophthalmology 2016; 123: 86-91.

45. Damato B. Progress in the management of patients with uveal melanoma. The 2012 Ashton Lecture. Eye 2012; 26: 1157-1172. 